\title{
Tangence
}

\section{Panorama, persistances et lignes de force : la critique québécoise en littérature française du $\operatorname{XIX}^{\mathrm{e}}$ siècle Panorama, persistencies and outlines: Quebec criticism on nineteenth-century French literature}

\section{Étienne Beaulieu}

Numéro 100, 2012

URI : https://id.erudit.org/iderudit/1017871ar

DOI : https://doi.org/10.7202/1017871ar

Aller au sommaire du numéro

Éditeur(s)

Tangence

ISSN

1189-4563 (imprimé)

1710-0305 (numérique)

Découvrir la revue

Citer cet article

Beaulieu, É. (2012). Panorama, persistances et lignes de force : la critique québécoise en littérature française du XIX ${ }^{\mathrm{e}}$ siècle. Tangence, (100), 65-77. https://doi.org/10.7202/1017871ar
Résumé de l'article

Si le Québec s'est très bien exporté culturellement dans les dernières décennies, surtout depuis la Révolution tranquille, on ne peut passer sous silence le fait qu'inversement, les chercheurs québécois ont aussi beaucoup contribué à faire du Québec une plaque tournante de l'activité de recherche dans nombre de disciplines, au premier chef desquelles on trouve forcément la littérature française et sa composante majeure que demeure celle du XIX ${ }^{\mathrm{e}}$ siècle. L'exercice de panorama fournit ici en ce sens l'occasion de tenter une désignation nouvelle de la réalité de la recherche québécoise sur la littérature $\mathrm{du} \mathrm{XIX}^{\mathrm{e}}$ siècle telle qu'elle se pratique. Mais il s'agira aussi de montrer l'effet inattendu et souvent inaperçu de persistances culturelles dans le choix effectué par certains chercheurs. Enfin, il s'agira de souligner que les dominantes méthodologiques du champ québécois en recherche sur le XIX ${ }^{\mathrm{e}}$ siècle français demeurent nettement l'histoire littéraire et les différentes composantes sociologiques et sociocritiques qui ont occasionné des discussions si vives ces dernières années dans le milieu universitaire québécois. 
Tangence $\square \mathrm{n}^{\circ}$ 100, 2012, p. 65-77.

\section{Panorama, persistances et lignes de force: la critique québécoise en littérature française du XIX ${ }^{\mathrm{e}}$ siècle \\ Étienne Beaulieu \\ Cégep de Drummondville}

En dressant un panorama de la critique québécoise portant sur la littérature française du XIX ${ }^{e}$ siècle, il semble difficile, voire impossible d'établir des critères sûrs qui permettraient de rendre compte avec justesse de l'activité réelle des chercheurs de ce domaine. L'ambiguïté de l'adjectif "québécois» est à elle seule hautement significative de l'état du champ littéraire et critique actuel: s'agitil de la recherche faite par des Québécois et publiée au Québec ou simplement de toute la recherche faite au Québec par des chercheurs de tous horizons ou encore de la recherche mise en œuvre par des Québécois, peu importe le lieu de sa publication? Dans le cadre de cet article, sera considéré comme québécois tout ce qui se rattache aux universités québécoises, qu'il s'agisse des chercheurs qui y ont été formés, des professeurs qui y ont enseigné ou qui y enseignent, des travaux qu'ils ont publiés ici ou à l'étranger, ou des structures de recherche qui y sont rattachées. Le simple fait de poser ces questions montre d'entrée de jeu que la recherche portant sur la littérature du $\mathrm{XIX}^{\mathrm{e}}$ siècle français n'est plus aussi aisément saisissable en ce qui concerne le champ québécois, à l'image d'ailleurs de la recherche portant sur les littératures des grands pays occidentaux et même sur les littératures postcoloniales. Celle du Québec a suivi la même voie en s'internationalisant toujours davantage, au point de montrer parfois un paradoxal surcroît d'activités en-dehors de ses frontières. On pense entre autres à tous les développements de la recherche 
québécoise en terres californiennes et italiennes, à Bologne plus précisément, ou encore en Pologne, à l'Université de Silésie où s'effectuent des recherches sur les littératures québécoises et canadiennes sous la direction de Krzysztof Jarosz ou du grand professeur Joseph Kwaterko. Si le Québec s'est très bien exporté culturellement dans les dernières décennies, surtout depuis la Révolution tranquille, on ne peut passer sous silence le fait qu'inversement, les chercheurs québécois ont aussi beaucoup contribué à faire du Québec une plaque tournante de l'activité de recherche dans nombre de disciplines, au premier chef desquels on trouve forcément la littérature française et sa composante majeure que demeure celle du XIX ${ }^{\mathrm{e}}$ siècle. Si Paris reste incontestablement la "capitale du $\mathrm{XIX}^{\mathrm{e}}$ siècle ${ }^{1}$ ", pour détourner la célèbre expression de l'essayiste germanophone Walter Benjamin, il n'en demeure pas moins vrai que le monopole de la recherche dans ce domaine tend à s'amenuiser dans le contexte très mouvant de la mobilité universitaire et collégiale d'aujourd'hui.

Le mot d'ordre de l'époque, en recherche comme en économie, semble en effet être celui de la mondialisation. Les échanges nationaux et internationaux, la mobilité transatlantique des professeurs en sont les signes, de même que la diversité des collaborations entre les grands centres de recherche de toute la francophonie et même hors de la francophonie - on pense entre autres aux développements majeurs des universités roumaines, polonaises et hongroises depuis leur entrée dans la zone euro, ou encore à tout le champ de la recherche tunisienne et japonaise, très importante par exemple dans la revue en ligne Flaubert ${ }^{2}$, hébergée par l'Université de Rouen et dirigée par Yvan Leclerc. L'ouverture des frontières critiques a probablement rendus caduques les termes par lesquels on pouvait dans le passé désigner par le moyen d'une expression claire tout ce champ d'activité. Si bien qu'un mouvement inverse commence à s'esquisser, du moins dans certains récents projets de recherche importants au Canada, qui font au contraire le pari du local. Ces projets proposent, comme c'est le cas par exemple des nouvelles chaires de recherche en littérature et en migration francophone dans l'Ouest canadien à l'Université de Saint-Boniface, de se concentrer sur la littérature locale dans des publications distribuées à moins vaste échelle, mais

1. Walter Benjamin, Paris, capitale du XIX ${ }^{e}$ siècle, Paris, Allia, 2003.

2. URL: http://flaubert.univ-rouen.fr/revue/ 
qui permettent de faire exister et connaître cette littérature à un public immédiat qui l'ignore souvent.

Quoi qu'il en soit, l'exercice du panorama (on sort difficilement du réseau des termes issus de son champ de recherche ${ }^{3}$ ) fournit en ce sens l'occasion de tenter une désignation nouvelle de la réalité de la recherche québécoise sur la littérature du XIx ${ }^{\mathrm{e}}$ siècle telle qu'elle se pratique. Il s'agira aussi de montrer l'effet inattendu et souvent inaperçu de persistances culturelles dans le choix effectué par certains chercheurs, comme c'est le cas par exemple des monographies consacrées au champ de la littérature du Xıx ${ }^{e}$ siècle français. Enfin, il faut mentionner tout de suite que les dominantes méthodologiques du champ québécois en recherche sur le XIX ${ }^{\mathrm{e}}$ siècle français demeurent nettement l'histoire littéraire et les différentes composantes sociologiques et sociocritiques qui ont occasionné des discussions si vives ces dernières années dans le milieu universitaire québécois. Si ces débats passionnés ont eu pour conséquence un certain clivage de la réalité pratique des chercheurs, ils ont en revanche permis l'émergence de nouveaux champs d'investigation et une transformation profonde des moyens par lesquels s'effectue la recherche, notamment en ce qui concerne la documentation et la diffusion des résultats, comme nous le verrons brièvement en fin de parcours à propos du tout nouveau site Medias19.org. Mais aussi et surtout, s'il faut déjà donner un aperçu des conclusions de cet article, le champ québécois montre que le découpage de la recherche en littérature par le moyen de la catégorisation par siècles hérité de Gustave Lanson et de l'histoire littéraire traditionnelle, pour commode qu'il demeure, montre néanmoins certains signes de remise en question. Enfin, soulignons tout de suite que le débat ayant eu lieu au Québec autour des questions de méthode a eu pour effet de décloisonner considérablement le champ chronologique des études sur le $\mathrm{xIx}^{\mathrm{e}}$ siècle et pour conséquence que les principaux et plus éminents chercheurs ne se cantonnent pour la plupart plus dans les limites arbitraires imposées par cette méthode

3. Le "panorama», qui s'est d'abord appelé le «diorama», est en effet un dispositif pictural et scénique "qui remonte aux dernières années du XviII ${ }^{\mathrm{e}}$ siècle» et qui s'est progressivement perfectionné au $\mathrm{XIX}^{\mathrm{e}}$ siècle jusqu'à devenir ce que ses contemporains appelaient une «salle des miracles ", grâce à son gigantesque effet réaliste en trompe-l'œil (Dolf Sternberger, Panoramas du XIX ${ }^{e}$ siècle [1974], trad. de l'allemand par Jean-François Boutout, Paris, Gallimard, coll. «Le promeneur», 1996, p. 15). 
demeurée pratiquement inchangée depuis plus d'un siècle quant aux fondamentaux de la recherche. La littérature du XIX siècle français demeure dans ce contexte un creuset et un bassin de références pour un nombre impressionnant de chercheurs, mais constitue de moins en moins un champ disciplinaire en soi $^{4}$.

\section{Persistances}

Si l'on consulte les monographies publiées au Québec, quelquesunes se révèlent très symptomatiques des voies empruntées par la recherche québécoise. C'est par exemple le cas des ouvrages de Cynthia Harvey (Université du Québec à Chicoutimi) portant sur la conception du roman de Théophile Gautier ${ }^{5}$, de Stéphane Vachon (Université de Montréal) sur Honoré de Balzac ${ }^{6}$, de Luc Bonenfant (Université du Québec à Montréal) sur le poète en prose Aloysius Bertrand $^{7}$ ou encore de Jean-François Richer (Université de Calgary) sur Honoré de Balzac ${ }^{8}$, de Maxime Prévost (Université d'Ottawa) sur Victor Hugo ou Alexandre Dumas ${ }^{9}$, et d'Étienne Beaulieu (Cégep de Drummondville) sur la pensée de Joseph Joubert ${ }^{10}$. Il faudrait

4. L’un des exemples les plus parlants est celui de Jean-François Hamel (Université du Québec à Montréal) qui a signé en 2006 peut-être l'essai le plus important paru récemment dans cet immense champ de recherche (Revenances de l'histoire. Répétition, narrativité, modernité, Paris, Minuit, coll. «Paradoxe», 2006), mais qui ne porte qu'en partie sur le XIx ${ }^{e}$ siècle. En effet, les figures de Victor Hugo, de Blanqui, de Jules Michelet ou de Baudelaire côtoient celles de Pierre Klossowski ou de Claude Simon. Les essais d'Isabelle Daunais, dont son plus récent par exemple (Les grandes disparitions. Essai sur la mémoire du roman, Saint-Denis, Presses universitaires de Vincennes, coll. «L'imaginaire du texte», 2008), traitent surtout de la question du roman traversant les siècles. La meilleure recherche en littérature du $\mathrm{XIX}^{\mathrm{e}}$ siècle français ne porte ainsi plus exclusivement sur cette période proprement dite.

5. Cynthia Harvey, Théophile Gautier, romancier romantique, Québec, Nota bene, 2007.

6. Stéphane Vachon, 1850, tombeau de Balzac, Montréal/Saint-Denis, XYZ/Presses universitaires de Vincennes, 2007.

7. Luc Bonenfant, Les avatars romantiques du genre. Transferts génériques dans l'œuvre d'Aloysius Bertrand, Québec, Nota bene, 2002.

8. Jean-François Richer, Les boudoirs dans l'œuvre d'Honoré de Balzac, Québec, Nota bene, 2012.

9. Maxime Prévost, Rictus romantiques. Politiques du rire chez Victor Hugo, Montréal, Presses de l'Université de Montréal, coll. "Socius», 2002 (prix du meilleur livre de l'APFUCC, 2004). Maxime Prévost et Yan Hamel (dir.), Victor Hugo 2003-1802: images et transfigurations, Montréal, Fides, 2003.

10. Étienne Beaulieu, La fatigue romanesque de Joseph Joubert, Québec, Presses de l'Université Laval, 2007. 
adjoindre à cette liste au moins le collectif publié par Luc Bonenfant, Marie-Andrée Beaudet (Université Laval) et Isabelle Daunais (Université McGill) qui s’intéresse aux figures marginales ou négligées du romantisme ${ }^{11}$.

Au sein de ces études, on note tout d'abord la prédominance de la recherche portant sur le genre romanesque: celle-ci dépasse de beaucoup ce qui s'est produit dans tout autre genre littéraire du $\mathrm{XIX}^{\mathrm{e}}$ siècle, comme l'essai (Joseph Joubert) ou la poésie (Aloysius Bertrand). Les raisons et motivations profondes de cet état de fait ne sauraient s'expliquer simplement par l'existence de grands groupes de recherche portant sur le roman, comme on en trouve à l'Université McGill, nommément le TSAR (Travaux sur les Arts du Roman) sous la direction d'Isabelle Daunais, ou encore par l'existence de grands regroupements de chercheurs dont les travaux portent sur la sociologie de la littérature ou la sociocritique, qui ont toujours eu des accointances particulières avec le monde romanesque, depuis les fondateurs du genre que sont Georges Lukács ou Lucien Goldmann ${ }^{12}$. Disons pour faire court que la forte présence de ces projets dans le champ québécois n'explique rien en ce qui concerne la prédominance du roman et qu'au contraire il faudrait expliquer leur prépondérance en regard de leur développement au Québec. Cette question exigerait à elle seule un très long argumentaire qui déborderait largement le cadre de cet article, mais disons pour aller rapidement qu'il semble que la progression fulgurante de l'intérêt pour le roman comme genre littéraire et l'explosion de toutes les variantes de l'investigation sociologique aient un lien direct avec la situation particulière du Québec en terre d'Amérique, lieu culturel accueillant des traditions si diverses et qui constitue un laboratoire de rêve pour les sociologues. Dans cette perspective, le Québec opère à la manière du roman qui reçoit des discours hétérogènes et tente de les changer en une œuvre cohérente ${ }^{13}$. Tout ceci est évidemment dit trop rapidement, mais il semble indéniable que si les causes doivent

11. Luc Bonenfant, Marie-Andrée Beaudet et Isabelle Daunais, Les oubliés du romantisme, Québec, Nota bene, 2004.

12. Georges Lukács, Théorie du roman, Paris, Denöel, 1968; Lucien Goldmann, Pour une sociologie du roman, Paris, Gallimard, coll. «Bibliothèque des idées», 1964.

13. Pour toutes ces questions d'ordre théorique, je renvoie à: Mikhail Bakhtine, Esthétique et théorie du roman [1978], préface de Michel Aucouturier, trad. du russe par Daria Olivier, Paris, Gallimard, 1987. 
être encore mieux expliquées, les effets de ce phénomène culturel demeurent parfaitement visibles dans la convergence et la domination presque sans partage des recherches portant sur le roman et la sociologie, à tout le moins en ce qui concerne la littérature du XIX ${ }^{e}$ siècle en terres québécoises.

Il faut souligner subséquemment que les monographies consacrées à des auteurs du XIX ${ }^{\mathrm{e}}$ siècle français par les chercheurs mentionnés précédemment touchent surtout le monde romantique et des figures que l'on pourrait qualifier soit d'importantes, voire de majeures, comme c'est le cas de Théophile Gautier, d'Honoré de Balzac, de Victor Hugo et d'Alexandre Dumas, soit, à tout le moins, de figures littéraires ayant conquis dans les dernières décennies une importance renouvelée ou carrément nouvelle, comme c'est le cas d'Aloysius Bertrand, de plus en plus pris en compte dans le champ des études sur les questions reliées aux genres littéraires et en particulier au poème en prose. Ou encore de Joseph Joubert qui, depuis son insertion dans la collection blanche chez Gallimard ${ }^{14}$, fait désormais figure d'auteur autrefois négligé, mais aujourd'hui lu attentivement par tous les spécialistes et même par certains écrivains n'ayant aucun rapport immédiat avec le champ universitaire, comme par exemple le romancier américain Paul Auster, traducteur et commentateur essayistique de Joubert ${ }^{15}$. C'est donc dire que bien des chercheurs québécois ont choisi en très grande part comme premier ouvrage ou comme champ de recherche stable et durable, l'œuvre d'un auteur romantique important, soit dans la tradition dix-neuviémiste, soit dans le champ des spécialistes de cette littérature.

14. Joseph Joubert, Carnets, éd. Jean-Paul Corsetti, Paris, Gallimard, 1994.

15. Il faut aussi dire que Joseph Joubert a eu droit dernièrement à un numéro de consécration dans la prestigieuse revue Europe ( ${ }^{\circ}$ 983, mars 2011, sous la direction d'Ariane Lüthi) et que plusieurs thèses de doctorat lui sont actuellement consacrées, notamment celle de Pierre Aussudre à l'Université Paris 3. Je contribue pour ma part à sa reconnaissance, tant en France (Étienne Beaulieu, «La prose du monde contre le roman. Joseph Joubert 1754-1824», Le Magazine littéraire, 2011, p. 98-100) qu'au Québec (Étienne Beaulieu, «Pourquoi faut-il lire Joubert?», Argument, vol. 12, nº 2, 2010, p. 156-162). La société des amis de Joseph Joubert vient par ailleurs de renaître de ses cendres, grâce à Jean-Luc Dauphin qui a aussi ressuscité la série des actes de colloque portant sur l'œuvre de Joubert (Jean-Luc Dauphin et Ariane Lüthi (dir.), Actes du $4^{e}$ colloque Joseph Joubert, Villeneuve-sur-Yonne, Les amis de Joseph Joubert, 2011). 


\section{Lignes de force}

Comment expliquer cette concentration de la recherche autour du romantisme et des grandes figures littéraires? Est-ce une trace, lointaine mais encore prégnante, de l'histoire littéraire québécoise marquée par l'influence des écrivains romantiques sur la critique, influence très puissante au XIX ${ }^{e}$ siècle et peut-être jusqu'à la Deuxième Guerre mondiale et sans doute même au-delà dans le cursus du cours classique, le phénomène allant s'atténuant avec le passage aux cursus publics dans les années $1960{ }^{16}$ ? Ensuite, sans pouvoir trancher catégoriquement ou simplement répondre à cette question, force est de constater que les ouvrages consacrés à la littérature du XIX ${ }^{e}$ siècle français par d'autres chercheurs, dont certains ont été formés en Europe (surtout en Belgique), aux États-Unis ou ailleurs dans le monde, concernent des écrivains du XIX ${ }^{e}$ siècle issus d'autres mouvements. C'est le cas par exemple des recherches de Véronique Cnockaert (Université du Québec à Montréal) qui sont surtout tournées vers la seconde moitié du XIX ${ }^{e}$ siècle et peut-être plus vers le Second Empire et la Troisième République, c'est-à-dire vers Émile Zola et le monde naturaliste, ou encore de celles de Pierre Popovic (Université de Montréal) qui choisit de son côté, dans son ouvrage le plus récent, de faire porter sa critique sur un personnage mineur du Second Empire ${ }^{17}$, dans le but de montrer à l'œuvre sa pensée, qualifiée de «folle» par plusieurs, et de renverser la perspective en démontrant tout ce que cet écrivain révèle de ce moment clé de l'histoire littéraire. C'est encore le cas des recherches d'Anthony Glinoer (Université de Sherbrooke), titulaire de la Chaire de recherche du Canada sur l'histoire de l'édition et la sociologie du littéraire, dont les travaux portent, certes, sur le monde romantique, mais dans une perspective qui contourne la canonisation littéraire en prenant pour objet l'édition romantique ou encore ce qu'il nomme «la littérature frénétique ${ }^{18}$ ». Il faut évidemment mentionner le cas

16. Sur ces questions, je me permets de renvoyer aux entrées de dictionnaire que j'ai consacrées soit directement à ce phénomène, soit aux auteurs du XIX ${ }^{\mathrm{e}}$ siècle québécois ayant subi l'influence du romantisme français, de manière contemporaine ou en décalage d'une ou plusieurs décennies (Alain Vaillant (dir.), Dictionnaire du romantisme, Paris, CNRS, 2012).

17. Pierre Popovic, Imaginaire social et folie littéraire. Le second Empire de Paulin Gagne, Montréal, Presses de l’Université de Montréal, coll. «Socius», 2008.

18. Anthony Glinoer reprend dans le titre de son ouvrage un adjectif déjà consacré à propos du romantisme «noir». Anthony Glinoer, La littérature frénétique, 
de Marc Angenot (Université McGill), titulaire des chaires JamesMcGill et Chaïm-Perelman de rhétorique et histoire des idées à l'Université Libre de Bruxelles, dont la voie tracée depuis des décennies dans le champ de l'histoire des idées vise explicitement à valoriser d'autres manières d'étudier le XIX $x^{e}$ siècle que celle des canons littéraires, notamment par le moyen de l'étude du discours social permettant de décloisonner le champ littéraire. Dans tous ces cas de figure, il semble que la formation littéraire en terres québécoises ait eu pour effet dominant, mais non pas exclusif il va sans dire, de tourner les chercheurs vers de grandes figures du monde romantique et que la formation européenne ou américaine en ait détourné la majeure partie ou, à tout le moins, ait tracé un chemin de contournement des grandes figures romantiques, soit par la voie de l'étude du roman, soit par les méthodes d'analyse provenant du champ des sciences sociales.

Il faut évidemment mentionner quelques exceptions notables, comme celle par exemple de Geneviève Sicotte (Université Concordia), spécialiste de la littérature naturaliste et symboliste et de la seconde moitié du siècle en général, de même que de différentes questions reliées à la sociologie de la littérature, comme celle de la représentation de la table ${ }^{19}$. C'est aussi le cas de Guillaume Pinson (Université Laval), spécialiste des sociabilités littéraires dont les recherches, d'abord centrées sur le monde aristocratique proustien, se tournent maintenant vers le monde de la presse imprimée du XIX ${ }^{e}$ siècle (sur lequel je reviendrai plus loin), dans le sillage de Marie-Ève Thérenty (Université Montpellier III) et d'Alain Vaillant (Université Paris-Ouest, Nanterre). Il faut mentionner aussi les recherches d'Isabelle Daunais (Université McGill), d'abord spécialiste de Gustave Flaubert, mais qui a infléchi significativement son parcours depuis quelques années vers les questions reliées au genre romanesque débordant le cadre strict des études dix-neuviémistes. Enfin, Geneviève Lafrance (Université du Québec à Montréal) offre un cas unique, hybride entre la spécialisation centrée sur un auteur,

Paris, Presses universitaires de France, coll. «Les littéraires», 2009; La querelle de la camaraderie littéraire. Les romantiques face à leurs contemporains, Genève, Droz, coll. «Histoire des idées et critique littéraire», nº 441, 2008; (avec Pascal Durand), Naissance de l'Éditeur. L'édition à l'âge romantique [2005], préface de Hubert Nyssen, Paris/Bruxelles, Les Impressions nouvelles, coll. "Réflexions faites», 2008.

19. Geneviève Sicotte, Le festin lu. Le repas chez Flaubert, Zola et Huysmans [1999], Montréal, Liber, coll. «Petite collection Liber», 2008. 
Madame de Staël en l'occurrence, et des questions d'ordre plus sociologique ou, ici, touchant plus à la philosophie de l'économie à l'époque romantique naissante ${ }^{20}$.

Mais si ces exceptions semblent faire pencher soudain la balance des chercheurs québécois vers la seconde moitié du siècle ou vers d'autres intérêts que les grandes figures romantiques, il faut nuancer cette affirmation. Geneviève Lafrance est aussi spécialiste de la grande figure romantique qu'est Madame de Staël. En outre, Guillaume Pinson et Geneviève Sicotte ont été formés dans le réseau de Marc Angenot à l'Université McGill, tandis qu'Isabelle Daunais, elle aussi de McGill, a reçu beaucoup de Jacques Neefs et de sa formation européenne aux tout débuts de sa carrière. Ce bilan se poursuivra maintenant, non du point de vue des corpus choisis, mais surtout par l'angle méthodologique adopté, notamment en ce qui concerne les questions sociales qui ont été ces dernières années au cœur des débats dans l'univers des dix-neuviémistes québécois.

\section{Questions de méthodes}

Le champ des études dix-neuviémistes a toujours été propice à l'éclosion de méthodes nouvelles portant sur les questions théoriques. On pense par exemple à l'interprétation biographique des œuvres par Charles Augustin Sainte-Beuve, dont la vision littéraire renaît aujourd'hui grâce aux recherches de José-Luis Diaz, président de la Société des études romantiques et dix-neuviémistes (SERD) et professeur à Paris viI-Denis-Diderot ${ }^{21}$. Les travaux de ce dernier n'ont étonnamment pas encore fait école au Québec, ce qui tend à confirmer un certain brouillage des voix parisiennes en sol québécois, tant est grande la portée universitaire de ce personnage dans le champ de la recherche hexagonale sur la littérature du $\mathrm{XIX}^{\mathrm{e}}$ siècle français. Cet étrange trou dans le champ des influences sur l'axe Paris-Québec s'explique peut-être en partie par le fait que les études balzaciennes étaient déjà très développées grâce aux travaux de Stéphane Vachon, professeur à l'Université de Montréal ${ }^{22}$.

20. Geneviève Lafrance, Qui perd gagne. Imaginaire du don et Révolution française, Montréal, Presses de l'Université de Montréal, coll. «Socius», 2008.

21. Voir, notamment, José-Luis Diaz, L’homme et l'œuvre, Paris, Presses universitaires de France, 2012.

22. Stéphane Vachon a par exemple publié, dirigé ou codirigé Les travaux et les jours d'Honoré de Balzac (P. du CNRS/PUV/PUM, 1992); Balzac. Une poétique 
Récemment, l'activité de recherche portant sur Balzac s'est d'ailleurs développée plus largement en terres canadiennes, depuis que Jean-François Richer (Université de Calgary), spécialiste des lieux dans l'univers de Balzac, publie lui aussi sur ce sujet ${ }^{23}$ après avoir terminé sa thèse sous la direction de Stéphane Vachon. La présence d'un universitaire de la carrure de Michel Pierssens au Québec, lui aussi professeur à l'Université de Montréal, a aussi une incidence marquée. L'importante revue Histoires littéraires, dont il est codirecteur, rayonne ainsi vers l'international, et notamment vers la France, à partir de Montréal, où s'effectue en grande partie le travail de publication menant à la diffusion de la revue. Notons au passage que cette revue se consacre autant à l'histoire littéraire du $\mathrm{XIX}^{\mathrm{e}}$ que du $\mathrm{xx}^{\mathrm{e}}$ siècle, ce qui tend à confirmer la thèse du décloisonnement avancé des siècles chez nombre d'importants chercheurs contemporains.

Mais ce n'est pas tant en histoire littéraire que les débats ont fait rage ces dernières années au Québec qu'en sociologie et sociocritique de la littérature. Il faut mentionner bien distinctement les deux termes, car c'est précisément de leur différence qu'il s'agit dans la querelle qui a opposé et oppose encore les tenants d'une analyse des institutions et de l'inscription du texte dans son contexte et les partisans de la lecture plus serrée de la présence du contexte dans le texte. Tout ce débat qui a secoué le monde des dix-neuviémistes québécois a mené à la fondation de ce que Yan Hamel et Olivier Parenteau ont appelé «l'École de Montréal»:

Au cours des dernières années, des chercheurs se réunissaient dans le cadre des activités organisées par le Collège de sociocritique de Montréal, qui s'est au fil du temps orienté vers un amalgame de théories d'obédience bourdieusienne où se mixent analyse des champs littéraires et des postures d'écrivains, histoire culturelle

du roman (PUV/XYZ, 1996); Itinéraires du dix-neuvième siècle II avec Roland Le Huenen (Centre d'études du XIX ${ }^{\mathrm{e}}$ siècle Joseph Sablé, 2001), et Réflexions sur l'autoréflexivité balzacienne avec Andrew Oliver (Centre d'études du XIX siècle Joseph Sablé, 2002). Il est aussi l'auteur d'un Balzac dans la collection «Mémoire de la critique» (P. de l'Université de Paris-Sorbonne, 1999), de Le Dernier Balzac (Du Lérot, 2001), de Les rivalités d'Honoré de Balzac. Analyses et documents (Société des amis de Balzac, 2007), de 1850. Tombeau d'Honoré de Balzac (XYZ/ PUV, 2007) et de À l'écoute du jeune Balzac. L'écho des premières æuvres publiées (1822-1829) avec Roland Chollet (Lévesque éditeur/PUV, 2012).

23. Voir Jean-François Richer, Les boudoirs dans l'œuvre d'Honoré de Balzac, ouvr. cité. 
classique, recours aux biographies d'éditeurs et d'auteurs, études des réseaux et des sociabilités, etc. Refusant de subordonner la lecture des textes à un conglomérat d'approches relevant moins des études littéraires et de l'analyse du discours que de l'histoire culturelle et de la sociologie empirique, et craignant que la sociocritique continue à être confondue avec ce qu'elle n'est pas jusqu'à en être menacée de dissolution, les collaborateurs de ce dossier ont récemment résolu de fonder le Centre de recherche interuniversitaire en sociocritique des textes (CRIST), qui doit, du moins l'espèrent-ils, leur permettre de faire rayonner l'esprit de «l'École de Montréal $»^{24}$.

Cette nouvelle école critique se veut ainsi à la fois implantée solidement dans la culture montréalaise, tout en étant ouverte aux travaux qui se font à l'étranger, selon une double perspective familière aux chercheurs québécois en littérature française du Xıx siècle. L'enjeu consiste en une coupure radicale qui séparerait les chercheurs tentés par tout ce qui borde la littérature et ceux qui craignent que le fait littéraire se noie dans la sociologie. Le Collège de sociocritique de Montréal s'est ainsi scindé en plusieurs groupes rassemblés autour de deux pôles importants: l'un plus strictement axé sur la sociocritique des textes (le CRIST) et l'autre plus largement ouvert aux approches de sociologie de la littérature (notamment la Chaire de recherche du Canada sur l'histoire de l'édition et la sociologie du littéraire et le site Internet Médias 19).

\section{Diffusion de la recherche}

Dans l'agitation créée par tous ces débats, peu de chercheurs ont aperçu que l'Association canadienne d'études francophones du $\mathrm{XIX}^{\mathrm{e}}$ siècle (ACEF XIX), qui tient encore son congrès annuel dans une ville canadienne sous l'égide des sociétés savantes (Fedcan), a perdu son organe de publication, les Cahiers $d u X_{X I X}{ }^{e}$ siècle. De 2006 à 2010, les Cahiers, fondés par Michel Pierssens et Maxime Prévost, auront donc paru cinq fois, cessant toute publication pour des raisons qui demeurent liées à la difficulté de la recherche en littérature française hors de France. Difficile en effet de maintenir un bon nombre d'abonnements dans ce contexte et seulement grâce à

24. Yan Hamel et Olivier Parenteau, «Pour la sociocritique: l'École de Montréal», Spirale, $\mathrm{n}^{\circ} 223$, novembre-décembre 2008, p. 15. 
la poste traditionnelle, à Internet et à la tenue d'un seul colloque annuel. L'orientation de ces Cahiers semblait de tendance nettement socio-historique, comme en témoigne la présence très forte d'historiens, tels Sylvain Venayre (Paris I-Panthéon-Sorbonne) ou Nathalie Richard (Paris I-Panthéon-Sorbonne), dans de nombreux articles de cette jeune revue disparue prématurément.

Néanmoins, les travaux de tendance socio-historique en littérature du $\mathrm{XIX}^{\mathrm{e}}$ siècle trouvent une nouvelle vigueur par le moyen du très important site Internet Médias 19 qui poursuit sur Internet le travail de recherche novateur, et peut-être le plus important entrepris depuis quelques décennies, d'Alain Vaillant et Marie-Ẽve Thérenthy ${ }^{25}$. Dans la foulée des recherches en littérature liées à l'histoire culturelle prise au sens large et à l'histoire médiatique au sens restreint, ce site, codirigé par Guillaume Pinson et Marie-Ève Thérenty, entend rendre accessible au public et aux chercheurs des documents autrement difficiles d'accès. Voici l'explication sommaire du projet disponible sur le site:

\section{Qui?}

Médias 19 est une plateforme scientifique dirigée par Guillaume Pinson, professeur au Département des littératures de l'Université Laval (Québec), et par Marie-Ève Thérenty, professeur et chercheur au RIRRA 21 de l'Université Paul-Valéry (Montpellier 3). De nature collaborative, elle fédère un ensemble de chercheurs qui s'intéressent à la presse du XIX $x^{\mathrm{e}}$ siècle. Un comité scientifique international veille aux orientations stratégiques de Médias 19 et rend des avis sur les propositions de publication.

\section{Pourquoi?}

Le site vise à fédérer la recherche sur la culture médiatique et à offrir aux chercheurs, aux étudiants et au grand public un accès direct à des sources souvent difficiles d'accès ou obscures. Les éditions annotées permettent de ressaisir les textes anciens dans leur contexte; elles sont consultables aussi bien en ligne qu'en générant le fichier PDF correspondant. Les notices biographiques de journalistes sont extraites des éditions annotées ainsi que des

25. Marie-Ève Thérenty, Mosaïques. Être écrivain entre presse et roman (1829-1836), Paris, Honoré Champion, 2003; Marie-Ève Thérenty et Alain Vaillant, Presses et plumes. Journalisme et littérature au XIX ${ }^{e}$ siècle, Paris, Nouveau Monde, 2004; Alain Vaillant et Marie-Ève Thérenty, 1836. L'An I de l'ère médiatique, Paris, Nouveau Monde, 2001. 
activités de recherche des collaborateurs de Médias 19. Les dossiers scientifiques, composés d'articles et d'analyses de documents, permettent de faire le point sur des problématiques en lien avec la culture médiatique ${ }^{26}$.

Financé entre autres par le Fonds québécois de la recherche sur la société et la culture (FRQSC) et par l'Université Laval, ce projet témoigne d'un élan nouveau dans la recherche québécoise sur la littérature française du $\mathrm{XIX}^{\mathrm{e}}$ siècle, non seulement par l'ampleur des travaux prévus, mais aussi par le décloisonnement disciplinaire qu'implique le positionnement de la littérature dans l'univers des discours sociaux publiés à plus vaste échelle que celle du livre.

\section{Une discipline en mutation}

Ainsi, au terme de ce bref parcours, il apparaît que non seulement le cadre chronologique du $\mathrm{XIX}^{\mathrm{e}}$ siècle mais aussi le carcan disciplinaire en lui-même tendent à s'estomper pour les chercheurs québécois contemporains, en dépit d'initiatives comme Cahiers $d u$ $X I X^{e}$ siècle et Médias 19 dont les intitulés sont éloquents et malgré certaines persistances plus ou moins canoniques dans le domaine romantique. Sous les pressions conjuguées des transformations de la discipline historique, de la domination presque sans partage au Québec des disciplines sociales et de l'étude du roman, ce que l'on appelle «la littérature française du XIx ${ }^{e}$ siècle» semble aujourd'hui de moins en moins clair, à tout le moins beaucoup moins discernable que ce n'était le cas pour l'histoire littéraire traditionnelle. C'est néanmoins dans ces moments de crise disciplinaire que surviennent le plus souvent les réorientations les plus salutaires pour l'avenir d'un champ de recherche.

26. URL: http://www.medias19.org/index.php?id=165 